\title{
Using a Kinect Sensor to Design and Implement an In- Home Rehabilitation System
}

\author{
Zaid Abdulelah Mundher \\ Department of Computers Sciences \\ College of Computer Sciences and Mathematics \\ University of Mosul \\ Mosul, Iraq
}

\begin{abstract}
A stroke, which is a type of brain injury, often has negative effects on moving, talking, or thinking. Therefore, stroke victims should get in a physical rehabilitation process. The rehabilitation process is performed in a rehabilitation center. However, doing exercises at home is more convenient for patients and the cost is low. In this work, a mobile robot is used to develop a physical rehabilitation system based on a Kinect sensor. Moreover, the proposed system combined with a proposed person following system, so the robot is able to track and follow patients.
\end{abstract}

\section{Keywords}

Home Rehabilitation System, Kinect sensor, Stroke victims.

\section{INTRODUCTION}

According to [1], about 15 million people per year suffer from a stroke. A stroke, which is a type of brain injury, often has negative effects on moving, talking, or thinking. Therefore, people with stroke should get in a rehabilitation process and they should keep doing exercises such as moving, stretching and practicing good posture to prevent these negative effects $[2,3]$. In general, exercises can help to decrease side effects of strokes, and help strokes' victims to get back to their usual activities.

Typically, the rehabilitation process is performed in a rehabilitation center, which can be considered as an expensive option for patients. However, computers and robots could be used to implement alternative affordable solution that may be used at home to help stroke patients to gain their possibilities again [4]. In the past few years, computers and robots have been used to implement at-home stroke rehabilitation systems [5]. Meanwhile, using a Kinect sensor in different research areas has been growing rapidly. Moreover, the Kinect sensor has also been applied in the physical therapy rehabilitation field.

Typically, rehabilitation systems are used the Kinect sensor as a fixed camera, so the main goal of this work is to introduce a rehabilitation system that use a moving Kinect sensor. This goal was achieved by using a mobile robot. Therefore, the proposed rehabilitation system is combined with a proposed person following system, so the robot is able to track and follow patients.

\section{RELATED WORKS}

There are already some works that has been done on using technology to build physical rehabilitation systems for stroke victims. However, some of the previous work does not use a Kinect sensor, so body sensors must be attached to the patient's body in order to capture the patient' motion data[6]. In addition, there already exists papers use the Kinect sensor to help stroke victims.

Comparing with [7][8] which are introduced motor arms exercise, and motor legs exercise, this paper introduces different kind of exercises. Moreover, this work uses a mobile robot instead of using a Kinect sensor as a fixed camera. In [9], a system was designed based on a Kinect sensor to build a stroke rehabilitation system. The main aim of that game is to train dynamic posture control. However, in [3], the Kinect sensor is also used as a fixed camera.

In this work, a mobile robot is used to develop a physical rehabilitation system based on a Kinect sensor. Moreover, the proposed system combined with a proposed person following system, so the robot is able to track and follow patients. As a result, patients can do their exercises in any place at their home.

\section{TECHNOLOGICAL BACKGROUND}

\subsection{The Kinect Sensor}

The Microsoft Kinect, which was released in November 2010, is a set of sensors that was originally developed as an input device for the Xbox 360 gaming console (Fig.1). The Kinect can track the human body movements, so the player can interact with the game without using a controller; the body is the controller [10].

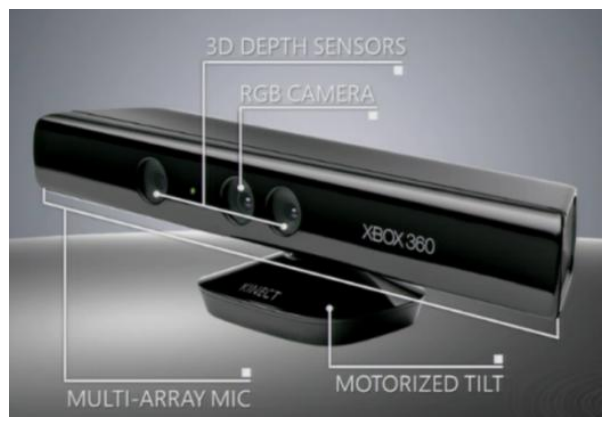

Fig.1: The Kinect sensor

The Kinect for Windows SDK is a toolkit that has a set of libraries to develop different applications for Kinect devices. Besides providing depth images, the Kinect for Windows SDK provides a skeleton-tracking feature that allows developers to recognize people and track their actions. Using depth sensors, the Kinect can recognize up to six users who are standing between 0.8 to 4.0 meters away (2.6 to 13.1 feet). Two of the detected users can be tracked in detail with twenty joint positions. Each skeleton joint is measured in a three dimensional $(\mathrm{X}, \mathrm{Y}, \mathrm{Z})$ plane. The $\mathrm{X}$ axis of the joint will change when the joints move from the right side to the left side or vice versa, while the $\mathrm{Y}$ axis will change when the 
joints move in the upwards or downwards direction. Similarly, $\mathrm{Z}$ axis will change when the joints move forwards or backwards in relation to the Kinect sensor [10]. The twenty joint points are shown in Fig.2.

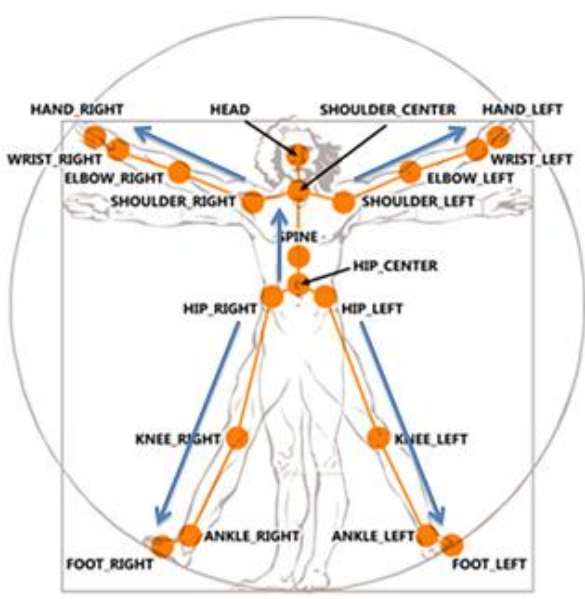

Fig.2: Skeleton joint points

\subsection{Human-Robot Interaction}

Speech recognition is one of the most important research areas in the field of the Human-Robot-Interaction. Since the proposed system is designed to work with patients people who prefer a simple and natural way to interact with robots, it was important to create a natural user interface to interact with the robot. Therefore, in this work, the Kinect sensor is used to develop and implement a speech recognition engine to provide a simple and natural interaction method through which patients people can send commands to the robot without the need for physical contact with the robot or the computer.

\section{THE PROPOSED SYSTEM}

An overview of the proposed system is shown in Fig.3.

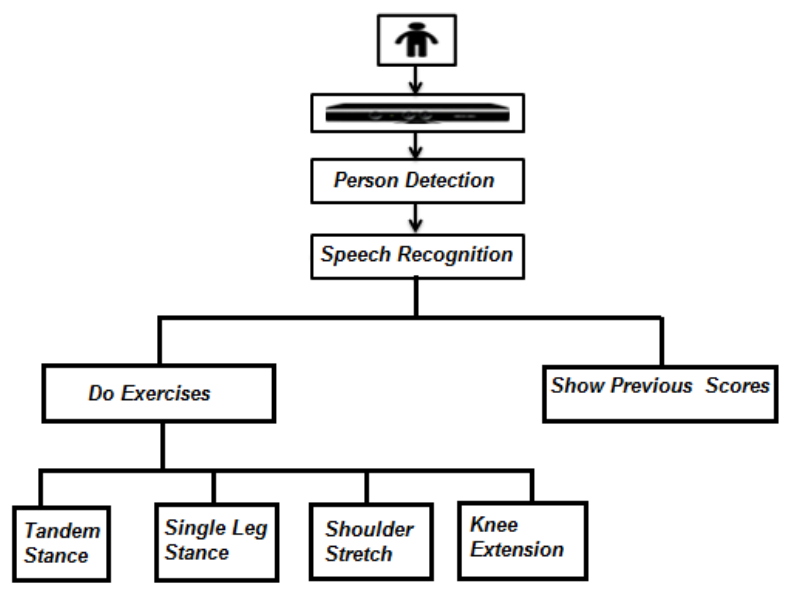

Fig.3: an overview of the proposed system

To build the proposed system, a long distance patient detection system needed to be implemented. Therefore, a Kinect sensor is used as an input device. To detect and track a patient, the skeleton stream from the Kinect is used. Once a patient's body is detected, the proposed algorithm starts processing the skeletal information.

The introduced system has two main types of exercises that a patient can choose from. These exercises, which have been taken from [11][12], are described below:

\subsection{Balance Exercises}

To improve the balance of the patients, the balance exercises were implemented. Two types of balance exercises were introduced: Tandem stance and single leg stance.

\subsubsection{Tandem Stance}

To achieve this exercise, a patient has to place one foot in front of the other, as Fig.4 shows.

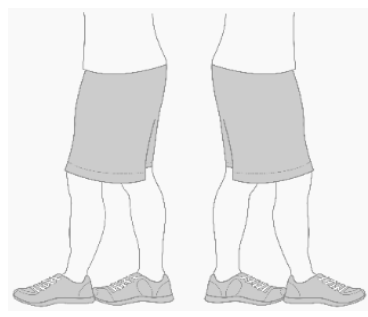

Fig.4: Tandem stance [13]

The algorithm of tandem stance is explained in the following: declare double xAnkleRight, xAnkleLeft

declare th $1=0.2$, th $2=0.04$;

$x$ AnkleRight $\leftarrow x$ coordinate of the skeleton AnkleRight point $x$ AnkleLeft $\leftarrow x$ coordinate of the skeleton AnkleLeft point

if(abs(xAnkleRight $-x$ AnkleLeft $)>t h 1)$

and abs(xShoulderRight $-x$ ShoulderLeft $)<t h 2)$

start timing

save scores

else

display the time

\subsubsection{Single Leg Stance}

This exercise is achieved by lifting on foot off the floor, as shown in Fig.5

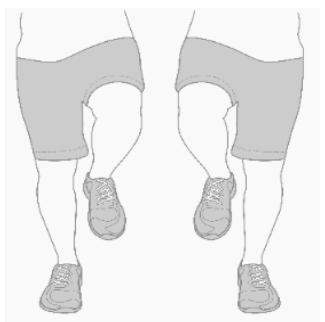

Fig. 5: Single leg stance [14]

The algorithm of single leg stance is explained in the following:

//Right Leg Stance

declare double yAnkleRight, yAnkleLeft

$y$ AnkleRight $\leftarrow y$ coordinate of the skeleton AnkleRight point

$y$ AnkleLeft $\leftarrow$ y coordinate of the skeleton AnkleLeft point

if $(y$ AnkleRight $+(-0.03)<y$ AnkleLeft +0.3$)$

start timing

else 
save scores

display the time

//Left Leg Stance

declare double yAnkleRight, yAnkleLeft

$y$ AnkleRight $\leftarrow$ y coordinate of the skeleton AnkleRight point

$y$ AnkleLeft $\leftarrow y$ coordinate of the skeleton AnkleLeft point

if $(y$ AnkleLeft+(-0.03) $<y$ AnkleRight $)$

start timing

else

save scores

display the time

\subsection{Stretching Exercises}

These exercises aim to help stroke victims to maintain a good range of movement in all joints and muscles. Two types of stretching exercises were introduced: shoulder extension and knee extension.

\subsubsection{Shoulder Stretch}

To achieve this exercise, a patient should stand facing a wall and place hands as high up on the wall as possible, as Fig.6 shows.

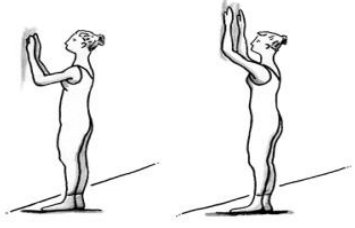

Fig.6: Shoulder stretch [15]

The algorithm of shoulder stretch is explained in the following:

declare double yHandRight, yHandLeft, yHead

declare th

$y$ HandRight $\leftarrow$ y coordinate of the skeleton HandRight point

$y$ HandLeft $\leftarrow y$ coordinate of the skeleton HandLeft point

$y$ Head $\leftarrow y$ coordinate of the skeleton Head point

if(yHandRight > yHead+0.1 and yHandLeft > yHead+0.1)

start timing

score $=\operatorname{abs}(y$ HandRight $-y H e a d)$

display score

save score

display time

save time

else

display score

display time

4.2.2 Knee Extension

This exercise is achieved by sitting in a chair; straighten out on leg, as shown in Fig.7
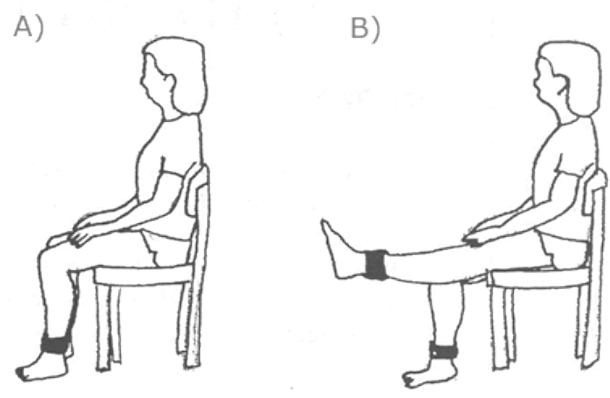

Fig.7: Knee extension [16]

The algorithm of the knee extension exercise is explained in following:

//Right knee extension

declare double yKneeRight, yKneeLeft, th=0.03

$y$ KneeRight $\leftarrow$ y coordinate of the skeleton KneeRight point

$y$ AngleRight $\square$ y coordinate of the skeleton AngleRight point

if (abs $(y$ KneeRight- yAngleRight $<t h))$

start timing

else

save time

display time

//Left knee extension

declare double yKneeLeft, yAnkleLeft, th

$y$ KneeLeft $\leftarrow y$ coordinate of the skeleton KneeLeft point

$y$ AnkleLeft $\leftarrow$ y coordinate of the skeleton AnkleLeft point

if $($ abs $(y$ KneeLeft $-y$ AnkleLeft $<$ th $))$

start timing

else

save time

display time

An xml file is created to store and restore the patient exercises scores. Moreover, a speech recognition engine was built based on the Windows SDK APIs. The main five commands that the introduced engine is able to recognize are described in Table 1 .

Table 1: Voice Commands

\begin{tabular}{|c|c|}
\hline Command & Description \\
\hline Tandem & Tandem stance exercise \\
\hline Leg_Right & Right leg stance exercise \\
\hline Leg_Left & Left leg stance exercise \\
\hline Shoulder & Shoulder extension exercise \\
\hline Knee_Right & Right knee extension exercise \\
\hline Knee_Left & Left knee extension exercise \\
\hline Scores & Display stored scores \\
\hline
\end{tabular}


In addition, the distance-based control loop approach is used to develop the proposed person-following subsystem. According to the proposed algorithm, the robot moves toward the target person when the distance is greater than $2 \mathrm{~m}$. Besides moving towards the target person, the robot needs to change its direction when the target person steps to the right side or left side. Therefore, the robot calculates the right and left motion parameters in order to keep the target person in the center of the robot's view. The proposed algorithm keeps tracking the right and left shoulders in order to determine the direction of the target person. Based on the shoulders' values, the robot will determine if a turn is necessary. As shown in Fig. 8(A), if the right and left shoulders are at the same distance from the robot (given a threshold), the forward command is executed. In addition, if the right shoulder is closer to the robot than the left shoulder, as shown in Fig. 8(B), the robot executes a rotate left command. If the left shoulder is closer to the robot from the right shoulder, as shown in Fig $8(\mathrm{C})$, the robot executes a rotate right command [17].

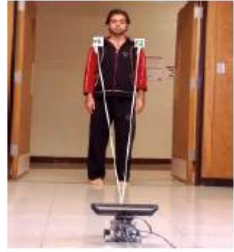

(A): Forward

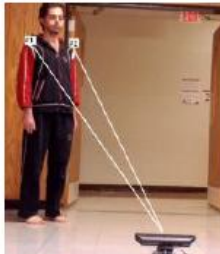

(B): Turn Left

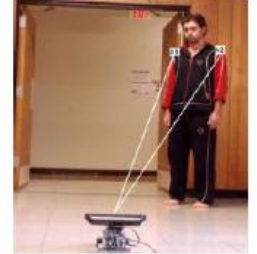

(C): Turn Right
Fig. 8: Person-following commands

\section{IMPLEMENTATION AND RESULTS}

The LEGO Mindstorm robot and the Kinect sensor are used to implement the proposed system. Kinect for Windows SDK with $\mathrm{C \#}$ is used to design the introduced system. The main user interface of the proposed system is shown in Fig. 9.

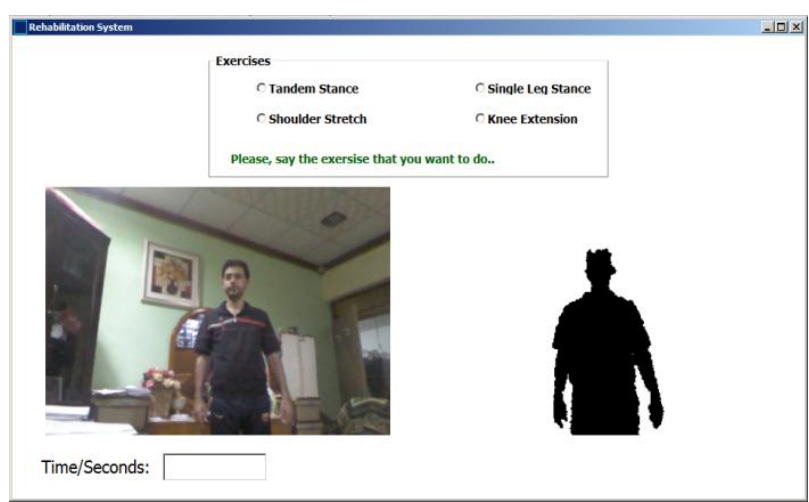

Fig.9: Main user interface

A human subject experiment was conducted to test the accuracy of the proposed system. Different human subjects were asked to stand in front of the robot and start doing exercises that are introduced by the proposed system.

Experiments were conducted in a real indoor environment. The results have shown that the proposed speech recognition system works perfectly, and the robot responds to the predefined commands. On the other hand, the results have also shown that the introduced person-following system works well, and the robot is able to move directly toward patients.

The result have shown the effectivity of the proposed system, where it is easy and comfortable to use.
The system was successfully tested in varying conditions. The performance of the proposed system is shown in Table 2 .

Table 2: Proposed rehabilitation system results

\begin{tabular}{|l|l|l|l|l|l|}
\hline Distance & $\begin{array}{l}\text { Tandem } \\
\text { stance }\end{array}$ & $\begin{array}{l}\text { One } \\
\text { leg } \\
\text { stance } \\
\text { stretch }\end{array}$ & $\begin{array}{l}\text { Shoulder } \\
\text { extension }\end{array}$ & $\begin{array}{l}\text { Knee } \\
\text { Accuracy }\end{array}$ \\
\hline 2.0 & & & & & $100 \%$ \\
\hline 2.5 & & & & & $100 \%$ \\
\hline 3.0 & & & & & $100 \%$ \\
\hline 3.5 & & & & & $100 \%$ \\
\hline
\end{tabular}

Based on the experimental results, and due to the Kinect hardware limitation, the proposed system cannot be run in the case of over 4 meters or less than 2 meters.

\section{CONCLUSION}

A Kinect sensor introduces a promising approach to address the rehabilitation process of the stroke victims. In this work, a Kinect sensor combined with a mobile robot was used to introduce a rehabilitation system for people with a stroke. The results have shown the effectivity of the Kinect sensor to design and develop these kinds of applications. Only four exercises have been implemented in this paper, and as a future work, a complete training course could be implemented.

\section{REFERENCES}

[1] King M, Hijmans JM, Sampson M, Satherley J, Hale L (2012) "Home-based stroke rehabilitation using computer gaming", New Zealand Journal of Physiotherapy 40(3): 128-134..

[2] US Agency for HealthCare Research and Quality, 1995,"Recovering After a Stroke: A Patient and Family Guide".

[3] Nicolae Palade, 2013, "Autonomous Stroke Rehabilitation with Microsoft Kinect".

[4] Gama, A., Teichrieb, V., Navab, N. , "Motor Rehabilitation Using Kinect:A Systematic Review", Games For Health Journal, V. 4, No, 2, 2015

[5] Chang, W. , Kim, Y.,"Robot-assisted Therapy in Stroke Rehabilitation", Journal of Stroke 2013;15(3):174-181.

[6] LaBelle, K., 2011 ,"Evaluation Of Kinect Joint Tracking For Clinical And In-Home Stroke Rehabilitation Tools".

[7] Lewis, C., Skelton, J., "Indexing and Retrieval for Xbox Kinect in-Home Rehabilitation System".

[8] Skelton, J., "Utilizing the Xbox Kinect to Help Facilitate At-Home Stroke Rehabilitation".

[9] Galna, B., Jackson, D, .. Rochester, L. , 2014 "Retraining function in people with Parkinson's disease using the Microsoft Kinect: game design and pilot testing",Journal of NeuroEngineering and Rehabilitaion".

[10] Miles, R. , Learn the Kinect API , O'Reilly Media, Inc., 2012

[11] Seguin, R., Epping, J., .. Nelson, M., "Growing Stronger: 
International Journal of Computer Applications (0975 - 8887)

Volume 171 - No. 6, August 2017

Strength Training for Older Adults", 2002.

[12] "Exercises for People with Parkinson's", Parkinson Society Canada, 2007.

[13] https://swaymedical.com/resources/protocols

[14] https://swaymedical.com/resources/protocols

[15] https://www.cancer.org/cancer/breastcancer/treatment/surgery-for-breast-cancer/exercises- after-breast-cancer-surgery.html

[16] http://www.keywordsuggests.com/ \%7C1kviBKd0LEft5V8sr\%7CfilbfMPHeJ*SoqBTrXnQ wTCE/

[17] Mundher, Z. , Zhong, J.,"A Real-Time Fall Detection System in Elderly Care Using Mobile Robot and Kinect Sensor", International Journal of Materials, Mechanics and Manufacturing, V. 2, No. 2, 2014 